\title{
Omega-shaped tag antenna with inductively-coupled feeding using U-shaped stepped- impedance resonators for RFID applications
}

\begin{abstract}
This study proposes a new omega-shaped tag antenna with inductively-coupled feeding (ICF) using U-shaped stepped- impedance resonators (SIRs). It aims at improving the performance of the tag antennas for Radio Frequency Identification (RFID) applications. The radiating body of the antenna is fed using two mirroring symmetrical U-shaped SIRs. This antenna is a simpler alternative for the existing antennas that match the impedance of the antenna to the chip impedance effectively applying varied reinforcement of the equivalent inductance of the radiating structure. In addition to the use of an omega-shaped structure, the proposed feeding technique boosts performance of the antenna impedance, dimensions, and peak gain. The measured size of the antenna was $50 \times 55.55 \times 1.6 \mathrm{~mm} 3$. It attains a peak gain of $1.8 \mathrm{dBi}$ and radiation efficiency higher than $85 \%$ at its operating frequency. The experimental results revealed that this tag antenna has the characteristic of good impedance matching within the frequency range of $900-940 \mathrm{MHz}$, corresponding to a better power reflection coefficient of -3 $\mathrm{dB}$. Comparison between the measured and simulated results verified that the proposed feeding method is capable to improve overall performance of RFID tag antennas.
\end{abstract}

